\title{
DEPENDENCE OF BILINEAR AND BIQUADRATIC INTERLAYER COUPLING ON THICKNESS OF MAGNETIC FILMS
}

\author{
J. BARnAŚ \\ Institute of Physics, A. Mickiewicz University \\ Matejki 48/49, 60-769 Poznań, Poland
}

\begin{abstract}
The dependence of bilinear and biquadratic interlayer coupling on the thickness of magnetic films is analysed for a trilayer structure with specular reflection at the outer surfaces. It is shown that the oscillation periods corresponding to the case where the thickness of one of the two magnetic films is constant, while that of the second one is varied, can be different from the oscillation periods in the case where the thicknesses of both magnetic films vary simultaneously. The nonoscillatory component of the coupling parameter is shown to be weakly dependent on the thickness of the magnetic films.
\end{abstract}

PACS numbers: 75.30.Et

Most experimental data on the interlayer coupling in magnetic multilayers can be explained by assuming the coupling energy $E_{c}$ in the form

$$
E_{\mathrm{c}}=-J_{1} \cos \varphi-J_{2} \cos ^{2} \varphi,
$$

where $\varphi$ is an angle between the magnetizations of the ferromagnetic films and $J_{1}$ and $J_{2}$ are the bilinear and biquadratic exchange parameters. The parameter $J_{1}$ oscillates in sign with increasing spacer thickness with one or more oscillation periods [1]. It also depends on the thickness of the magnetic films [2-5]. In the latter case $J_{1}$ consists of two terms, $J_{1}=J_{1}^{(\mathrm{n})}+J_{1}^{(\circ)}$, where $J_{1}^{(\mathrm{n})}$ and $J_{1}^{(\circ)}$ are respectively the nonoscillatory and oscillatory components. On the contrary, only negative values of the parameter $J_{2}$ were reported up to now. There are several physical mechanisms which can contribute to the biquadratic coupling [6]. In this paper, however, only the intrinsic mechanism [7] will be considered.

The oscillatory coupling is a result of spin dependent interference of electron waves reflected from interfaces and surfaces. Therefore, the coupling parameter depends significantly on the boundary conditions at the outer surfaces of the magnetic films. In this paper we consider the case where the electrons are specularly reflected at those surfaces. When considering the dependence of the coupling parameter on the thickness of the magnetic films in a sandwich structure, one has to distinguish between the case where the thickness of one of the two magnetic films 
is constant while the thickness of the second one is varied (i), and the case where both magnetic films have equal thicknesses which vary simultaneously (ii).

Consider two magnetic films of thicknesses $d_{1}$ and $d_{2}$, which are separated by a nonmagnetic metallic film of thickness $d_{0}$. The coupling parameters $J_{1}$ and $J_{2}$ can be calculated from the formulas

$$
\begin{aligned}
& J_{1}=\frac{1}{2}\left(E_{\uparrow \downarrow}-E_{\uparrow \uparrow}\right), \\
& J_{2}=E_{\perp}-\frac{1}{2}\left(E_{\uparrow \downarrow}+E_{\uparrow \uparrow}\right),
\end{aligned}
$$

where $E_{\uparrow \downarrow}, E_{\uparrow \uparrow}$ and $E_{\perp}$ are the total energies per unit area respectively in the antiparallel, parallel and perpendicular configurations of the film magnetizations. Assuming free-electron-like model one can easily calculate them from solutions of the appropriate Schrödinger equation with an effective spin dependent electron potential $U_{s}(z)$. In the spacer layer this potential is independent of the spin orientation, $U_{s}(z)=U_{0}$, whereas inside the magnetic films $U_{s}(z)=U_{+}$, if $s$ corresponds to the spin-majority electrons and $U_{s}(z)=U_{-}$otherwise. The Fermi level will be adjusted in such a way that the total number of electrons is the same as in the bulk limit corresponding to the chemical potential $\mu$. A more general description within the jellium model [8] will be presented elsewhere.

The dependence of the coupling parameter $J_{1}$ on the thickness of the magnetic films is shown in Fig. 1. The left column corresponds to the situation when the thickness of one of the magnetic films $\left(d_{1}\right)$ is fixed, while the thickness of the second magnetic film $\left(d_{2}\right)$ is varied (case (i)), whereas the right column corresponds to the case where both magnetic films have equal thicknesses, $d_{1}=d_{2}$ (case (ii)). In the strong coupling limit (Figs. 1a and 1e) the corresponding curves are qualitatively similar and in both cases the parameter $J_{1}$ consists of nonoscillatory and oscillatory parts. The nonoscillatory terms are generally thickness dependent, while the oscillatory components vary with increasing thickness of the magnetic films with the oscillation period $\Lambda_{+}=\lambda_{F+} / 2$.

Consider now what happens when $U_{-}$decreases, so both electron spin-subbands in the magnetic films are occupied. Two qualitatively new features appear now in the dependence of the parameter $J_{1}$ on the thickness of the magnetic films. The first one is the occurrence of an additional oscillation period, which originates from the spin-minority electron band. This is clearly evident in Fig. 1c, where one can note two different oscillation periods, $\Lambda_{+}=\lambda_{\mathrm{F}+} / 2$ and $\Lambda_{-}=$ $\lambda_{F-} / 2$. Here $\lambda_{F+}$ and $\lambda_{F-}$ are the Fermi wavelengths respectively for the spin-majority and spin-minority electrons. For the parameters assumed in Fig. 1c one finds $\Lambda_{+}=3.06 \AA$ and $\Lambda_{-}=2 \Lambda_{+}=6.12 \AA$. The second new feature is a split of each maximum in the case (ii) into two separate maxima (see Fig. 1f). The dips between those maxima increase with decreasing $U_{-}$, and for small values of $\Delta U=U_{-}-U_{+}$, one observes a split of the corresponding oscillation period, as it is clearly evident in Fig. $1 \mathrm{~h}$.

Similar features also occur in the dependence of the parameter $J_{2}$ on the thickness of the magnetic films, as shown by the solid lines in Figs. $2 \mathrm{a}$ and $2 \mathrm{~b}$, respectively for the case (i) and (ii). For comparison, the parameter $J_{1}$ is also shown there (dotted lines). One additional feature of the biquadratic coupling 


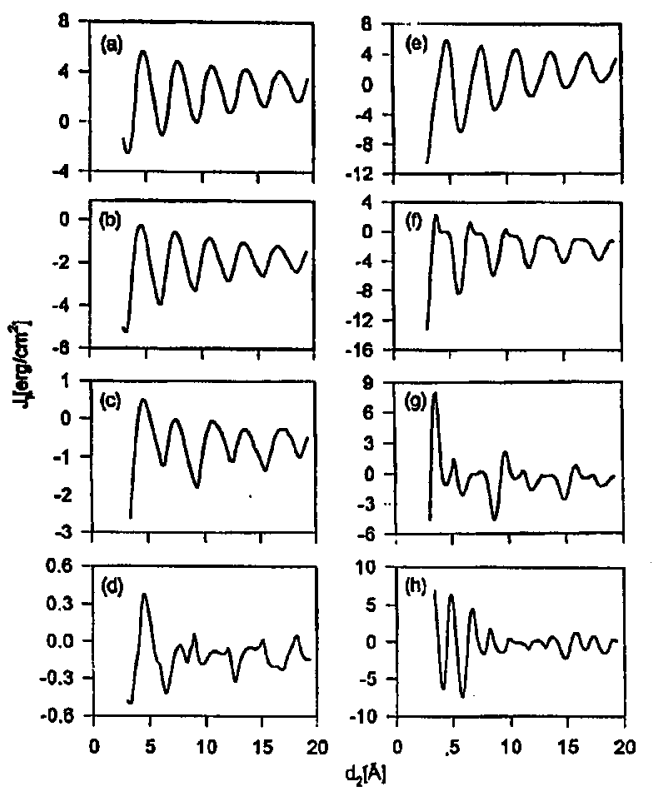

Fig. 1. Dependence of the bilinear coupling parameter $J_{1}$ on the thickness $d_{2}$ of the magnetic film. The left and right columns correspond respectively to the cases where the thickness of the second magnetic film is constant $\left(d_{1}=20 \AA\right)$ and varies simultaneously with $d_{2}\left(d_{1}=d_{2}\right)$. The parameters assumed in numerical calculations are: $\mu=4 \mathrm{eV}$, $U_{+}=U_{0}=0$ and $d_{0}=10 \AA$. The other parameters are: $U_{-}=6 \mathrm{eV}(\mathrm{a}, \mathrm{e}), U_{-}=4 \mathrm{eV}$ $(\mathrm{b}, \mathrm{f}), U_{-}=3 \mathrm{eV}(\mathrm{c}, \mathrm{g})$ and $U_{-}=2 \mathrm{eV}(\mathrm{d}, \mathrm{h})$.
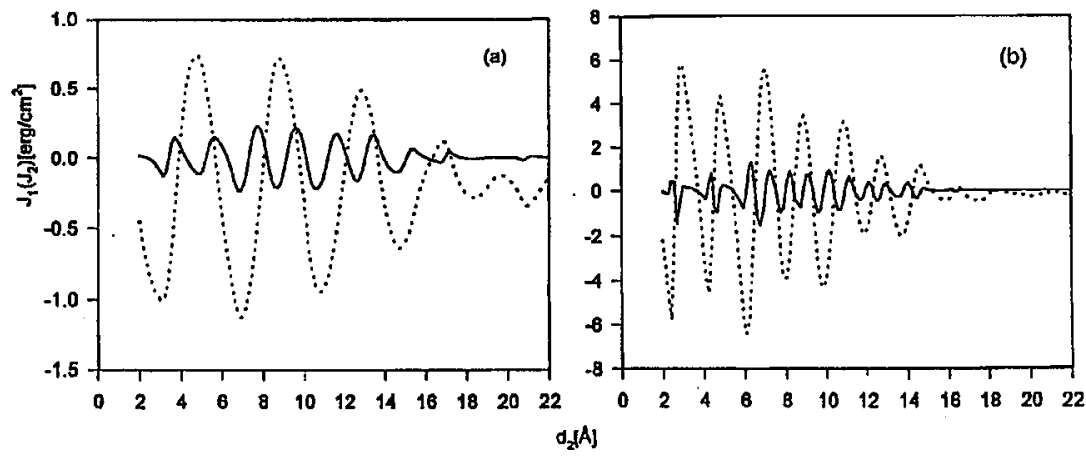

Fig. 2. Dependence of the biquadratic coupling parameter $J_{2}$ (solid lines) on the thickness $d_{2}$ of the magnetic film for (a) $d_{1}=30 \AA$ and (b) $d_{1}=d_{2}$. The other parameters are: $U_{+}=U_{0}=0, U_{-}=1 \mathrm{eV}, \mu=4 \mathrm{eV}$ and $d_{0}=5 \AA$. For comparison the corresponding parameter $J_{1}$ is also shown (dotted lines). 
is noteworthy. For asymmetrical structures the points where $J_{2}$ vanishes do not coincide generally with the nodes of $J_{1}$.

It is necessary to emphasize that the above predictions apply to trilayers with specularly reflecting outer boundaries and with the electron mean free path much longer than the total thickness of the structure.

\section{Acknowledgments}

The work has been carried out under the research project No. 2 P302 11606 of the Committee for Scientific Research.

\section{References}

[1] S.S.P. Parkin, in: Ultrathin Magnetic Structures II, Eds. B. Heinrich, J.A.C. Bland, Springer-Verlag, Berlin 1994, p. 148.

[2] P.J.H. Bloemen, M.T. Johnson, M.T.H. van de Vorst, R. Coehoorn, J.J. de Vries, R. Jungblut, J. aan de Stegge, A. Reinders, W.J.M. de Jonge, Phys. Rev. Lett. 72, 764 (1994).

[3] P. Bruno, Europhys. Lett. 23, 615 (1993).

[4] S. Krompiewski, J. Magn. Magn. Mater. 140-144, 515 (1995).

[5] J. d'Albuquerque e Castro, J. Mathon, M. Villeret, D.M. Edwards, Phys. Rev. B 51, 12876 (1995).

[6] J.C. Slonczewski, J. Appl. Phys. 73, 5957 (1993).

[7] J. Barnaś, P. Grünberg, J. Magn. Magn. Mater. 121, 326 (1993).

[8] W. Schmidt, J. Magn. Magn. Mater. 145, 181 (1995). 